\title{
Treatment options for refractory and difficult to treat seizures: focus on vigabatrin
}

\author{
This article was published in the following Dove Press journal: \\ Therapeutics and Clinical Risk Management \\ 3I August 201। \\ Number of times this article has been viewed
}

\author{
Justin A Tolman \\ Michele A Faulkner \\ School of Pharmacy and Health \\ Professions, Creighton University, \\ Omaha, Nebraska, NE, USA
}

Correspondence: Justin A Tolman School of Pharmacy and Health Professions, Creighton University, 2500 California Plaza, Omaha, NE 68178, USA

$\mathrm{Tel}+\mathrm{I} 4022802915$

Fax +I 4022801883

Email justintolman@creighton.edu

\begin{abstract}
Complex partial seizures are often refractory to current pharmacological therapies. These difficult to treat seizures are typically managed using multiple antiepileptic drugs (AEDs). AEDs as a group are frequently associated with significant adverse drug effects, multiple drug interactions, and numerous potential clinical complications due to their individual pharmacokinetic profiles and unique drug properties. Recently, the approval of vigabatrin by the US Food and Drug Administration has necessitated that clinicians re-evaluate these risk-benefit relationships and determine where the drug fits within the treatment scheme for the management of complex partial seizures. This review will facilitate that re-evaluation through a brief review of AEDs used in the treatment of complex partial seizures, followed by a focused discussion on vigabatrin.
\end{abstract}

Keywords: vigabatrin, complex partial seizures, refractory seizures, risk evaluation and mitigation strategy, epilepsy

\section{Introduction}

Seizures are typically categorized into several general classifications, ie, idiopathic, genetic (likely polygenic or oligogenic in origin), symptomatic (associated with inborn or acquired anatomical or pathological abnormalities), provoked (caused by a definite factor that is not anatomical or pathological in nature), or cryptogenic (suspected definite cause of unknown origin). These classifications include wide-ranging causes of seizure activity, such as chromosomal or gene-based abnormalities, structural aberrations in neuronal growth or spatial arrangement, infectious processes, traumatic injury, malignancies, metabolic abnormalities, cerebral hemorrhages or infarctions, or inflammatory processes. ${ }^{1}$ Seizure activity is further defined based on the seizure type(s), with additional information provided by the signs and symptoms of seizure activity and radiological or electroencephalographic findings. Recurrent unprovoked seizure activity usually results in a diagnosis of epilepsy.

The classifications and functional descriptions of seizure activity are used clinically to inform therapeutic decisions. The treatment of recurrent seizures predominantly centers on the utilization of one antiepileptic drug (AED), with possible adjunctive use of a second or even third agent in the case of monotherapeutic failure. The majority of patients with recurrent seizures are successfully managed through the use of a single AED. Some patients require successive monotherapeutic trials. However, approximately $30 \%-40 \%$ of epileptic patients have inadequate seizure control with just one AED, and require the use of adjunctive agents. ${ }^{2}$ A subset of this group will have regular and persistent seizure activity despite reasonable doses of multiple AEDs. 
These seizures are considered refractory to treatment. ${ }^{3}$ One of the most common refractory seizure types is complex partial seizures. ${ }^{4}$ These seizures arise in specific and localized cortical regions within one hemisphere and result in impaired consciousness. They involve a focal cerebral alteration with or without pathological specificity, have asymmetric symptoms associated with the site of seizure generation, are generally short in duration, and may evolve into secondary generalized seizures. Complex partial seizures dramatically impact quality of life measurements through functional and social limitations imposed by periodic and unanticipated impairments in consciousness.

Difficult to treat seizures are of particular concern due to the risks of increased morbidity and mortality. Epileptic patients have increased risks of accidental injuries, cerebral or cognitive damage, and psychiatric or psychosocial conditions. Epilepsy has also been linked to sudden unexplained death in epilepsy (SUDEP), accidental death, and suicide. ${ }^{5}$ However, AEDs themselves may cause serious adverse effects, including hepatic and/or renal toxicity, cardiac aberrations, blood dyscrasias, cognitive or psychiatric impairments, skin disorders, and vision changes. The therapeutic management of epilepsy, including difficult to treat seizures, has historically been undertaken to balance adequate seizure control with drug-related adverse effects. ${ }^{6}$ Recently, the approval of vigabatrin by the US Food and Drug Administration (FDA), has necessitated that clinicians re-evaluate these risk-benefit relationships, and determine where to place vigabatrin within treatment schemes for the management of complex partial seizures. This review will facilitate that re-evaluation through a brief review of AEDs used in the treatment of complex partial seizures, followed by a focused discussion on vigabatrin.

\section{Antiepileptic drugs}

Many AEDs have been approved as monotherapy and/or as adjunctive agents for the treatment of complex partial seizures (Table 1). This discussion will focus on the medications according to their FDA-approved indications, although data do exist to support the use of some agents that are approved as adjunctive therapy for use as monotherapies to treat complex partial seizures. Therapeutic plans are often generated based on individual disease and patient factors, and are influenced by the experience of clinicians rather than by following a proscribed treatment algorithm. AED activity against complex partial seizures is often studied in populations of treatmentrefractory patients, with efficacy measures generally reported as the percentage of patients with a $\geq 50 \%$ reduction in
Table I Indications of common antiepileptic agents approved by the US Food and Drug Administration for treatment of complex partial seizures

\begin{tabular}{ll}
\hline $\begin{array}{l}\text { Monotherapy } \\
\text { agents }\end{array}$ & $\begin{array}{l}\text { Adjunctive therapy } \\
\text { agents }\end{array}$ \\
\hline $\begin{array}{l}\text { Phenytoin } \\
\text { Valproic acid } \\
\text { Carbamazepine } \\
\text { Oxcarbazepine }\end{array}$ & Valproic acid \\
Phenobarbital & Oxcarbazepine \\
Topiramate & \\
Lamotrigine & Topiramate \\
Felbamate & Lamotrigine \\
& Felbamate \\
& Gabapentin \\
& Pregabalin \\
& Tiagabine \\
& Levetiracetam \\
& Zonisamide \\
& Lacosamide \\
& Vigabatrin \\
\hline
\end{tabular}

seizure activity, or as some time period with the absence of seizure activity (for example seizure-free periods of six, 12, or 24 months). ${ }^{7}$ These reported measures of efficacy would likely under-represent expected clinical antiseizure activity in a normal complex partial seizure patient population. The selection of antiepileptic drug regimens may be influenced by numerous factors, including seizure type, frequency, and intensity, expected response rates, adverse effect profiles, therapeutic drug monitoring requirements, possible drug interactions, patient age, neurological and cognitive functioning, comorbid conditions, renal and hepatic function, and measures of quality of life.

\section{Monotherapy agents for complex partial seizures Phenytoin}

Phenytoin has long been considered a first-line agent in the treatment of complex partial seizures. It has well documented antiseizure activity, with studies reporting 30\%-60\% response rates (Table 2) ${ }^{8,9}$ Despite strong efficacy results and widespread clinical acceptance, phenytoin might no longer be considered a first-line AED in some clinical practices due to its unusual pharmacokinetic properties, requirement for regular therapeutic drug monitoring, significant drug interactions, and problematic adverse drug effects.

Phenytoin is highly bioavailable, but with a slow and variable rate of absorption that leads to variable maximal drug concentrations $\left(\mathrm{C}_{\max }\right)$ and times observed to reach the $\mathrm{C}_{\max }\left(\mathrm{t}_{\max }\right)$. It is very highly bound to plasma proteins with a small free fraction of unbound drug, making therapeutic 
Table 2 Approximate antiseizure efficacy for the treatment of complex partial seizures using agents approved by the US Food and Drug Administration for use as monotherapy

\begin{tabular}{ll}
\hline Antiepileptic drug & $\begin{array}{l}\text { Approximate efficacy } \\
\text { (patients with } \geq \mathbf{5 0} \% \\
\text { decrease in seizure activity) }\end{array}$ \\
\hline Phenytoin & $30 \%-60 \%$ \\
Valproic acid & $20 \%-50 \%$ \\
Carbamazepine & $20 \%-40 \%$ \\
Oxcarbazepine & $20 \%-30 \%$ \\
Phenobarbital & $30 \%-40 \%$ \\
Topiramate & $20 \%-50 \%$ \\
Lamotrigine & $10 \%-30 \%$ \\
Felbamate & $*$ \\
\hline
\end{tabular}

Note: *Felbamate efficacy is reported as an absolute decrease in seizure activity and not as a percentage of patients with at least a $50 \%$ decrease in seizure activity.

adjustments necessary for patients with hypoalbuminemia and creating the potential for competitive plasma protein binding-based drug interactions. Adjustments to phenytoin regimens are typically based on successful seizure control, which typically occurs within a therapeutic range of $10-20 \mu \mathrm{g} / \mathrm{mL}$. Phenytoin is highly metabolized, with a widely variable drug elimination half-life due to saturable drug metabolism at therapeutic doses. Saturable drug metabolism is also problematic when perturbations to normal enzymatic functioning alter phenytoin concentrations, as occurs in numerous drug-drug interactions. Phenytoin regimens should be individualized based on clinical response and serum concentrations, with special precautions taken whenever adjusting drug doses. ${ }^{10}$

Phenytoin has an extensive list of side effects associated with short-term and chronic use. Adverse central nervous system (CNS) effects are prominent at therapeutic drug concentrations, and include dizziness, drowsiness, lethargy, headache, and nystagmus. Dystonia, seizures, coma, and death are possible as drug concentrations increase to the toxic $(>40 \mu \mathrm{g} / \mathrm{mL})$ or lethal $(>100 \mu \mathrm{g} / \mathrm{mL})$ range. Additional common adverse effects include cutaneous rashes, nausea, vomiting, and constipation. Idiosyncratic hypersensitivity reactions to phenytoin may consist of blood dyscrasias, including agranulocytosis, Hodgkin's lymphoma, and pancytopenia.

Dermatological abnormalities, such as exfoliative dermatitis, Stevens-Johnson syndrome, and toxic epidermal necrolysis, have also been observed. Phenytoin can induce cardiac abnormalities, such as arrhythmias, hepatitis, changes in blood glucose, and hypothyroidism. Chronic therapy has also been associated with behavioral changes, cognitive impairments, cerebellar ataxia, skin thickening, fat redistribution, gingival hyperplasia, hirsuitism, acne, and osteomalacia. ${ }^{1}$

\section{Valproic acid}

Valproic acid has antiseizure activity equivalent to that of phenytoin in the treatment of complex partial seizures, with similar response rates of approximately $20 \%-50 \%{ }^{8,11}$ It is commercially available in different forms, including the free acid form (valproic acid), its sodium salt (sodium valproate), and a coordinated sodium salt complex (divalproex sodium), each with slightly different formulations and pharmacokinetic properties, but with equivalent pharmacological effects. The extent of absorption is equivalent for all formulations, each having a bioavailability of approximately $100 \%$. Both food and the formulation affect the rate of drug absorption and can lead to differences in $\mathrm{C}_{\max }$ and $\mathrm{t}_{\max }$ values, with alterations of gastrointestinal $\mathrm{pH}$ affecting drug bioavailability. Valproic acid has saturable protein binding that produces nonproportional changes in drug effects and adverse effects at the upper range of therapeutic drug concentrations. A serum valproic acid concentration range of $50-100 \mu \mathrm{g} / \mathrm{mL}$ is typically associated with optimal antiseizure effects, and concentrations $>100-125 \mu \mathrm{g} / \mathrm{mL}$ have been associated with increased frequency and severity of adverse effects. Adequate seizure control is achieved in some patients at higher than normal concentrations, and efficacy and adverse effects should be monitored, with dose regimen adjustments made on a case-by-case basis. Valproic acid is extensively metabolized by hepatic enzymes other than those in the cytochrome P450 (CYP) system, with less than 3\% of the dose eliminated unchanged in the urine. It is involved in numerous drug interactions, the potential for which should be evaluated when starting valproic acid therapy or at the initiation, termination, or adjustment of other medications..$^{10}$

Valproic acid commonly induces gastrointestinal adverse effects, including nausea, vomiting, and abdominal pain, with the possibility for substantial weight gain in many patients. Adverse CNS effects are also common, and include tremors, headaches, somnolence, weakness, and dizziness, with rare reports of drug-induced encephalopathy. Serious hepatotoxicity and associated fatalities have been reported necessitating liver function testing, primarily at the initiation of therapy or with changes to a dosage regimen. Dermatological reactions have also been reported, including transient alopecia and skin rash, with rare reports of StevensJohnson syndrome. Valproic acid has been associated with dose-dependent thrombocytopenia and infrequent instances of bone marrow suppression. Rare reports of multiorgan 
system failure following initiation of valproic acid therapy also necessitate early monitoring and evaluation. ${ }^{1}$

\section{Carbamazepine}

Carbamazepine has also demonstrated good seizure control, with a $20 \%-40 \%$ response rate, and equivalent activity to phenytoin and valproic acid in the management of complex partial seizures. ${ }^{8}, 12$ The absorption of carbamazepine is slow and variable following oral administration, leading to variable $\mathrm{C}_{\text {max }}$ and $\mathrm{t}_{\text {max }}$ values. It has clinically insignificant protein binding, is substantially distributed throughout the body, and has cerebrospinal concentrations approximately equal to free drug concentrations in the blood, supporting a rationale for serum drug level testing.

Carbamazepine is extensively metabolized, and significantly induces its own metabolism over a period of 3-5 weeks. This metabolic autoinduction shortens the elimination half-life from 72 hours to $12-17$ hours, and necessitates dose adjustments, with a usual target therapeutic window of $4-12 \mu \mathrm{g} / \mathrm{mL}$. Carbamazepine has numerous clinically significant interactions with other AEDs, and medications should be evaluated when initiating therapy or making adjustments to this or other regimens. ${ }^{10}$

Commonly reported adverse effects of carbamazepine include neurosensory disturbances of vision, balance, and movement, as well as CNS effects, such as dizziness, headaches, confusion, nausea, and vomiting. On occasion, the drug may induce clinically relevant hyponatremia. Severe and potentially fatal cardiac disturbances have been rarely reported, including thromboembolic events, thrombophlebitis, development of arrhythmias and atrioventricular block, congestive heart failure, edema, hypotension, syncope, and cardiac arrest. Transient leukopenia is the most commonly reported hematological adverse event associated with carbamazepine, necessitating periodic assessment of white blood cell counts and possible drug discontinuation if levels fall below critical predefined thresholds. Additional blood disorders, including agranulocytosis, aplastic anemia, pancytopenia, and thrombocytopenia have been noted with carbamazepine use.

Dermatological reactions have been associated with carbamazepine use and include photosensitivity and rashes. Exfoliative dermatitis, Stevens-Johnson syndrome, toxic epidermal necrolysis, and acute generalized exanthematous pustulosis are rare but serious conditions also sometimes associated with carbamazepine therapy. ${ }^{1}$ It has recently been recommended that patients of Asian descent be tested for the presence of an allele encoding for a human leukocyte antigen (HLA-B*1502) associated with increased risk of Stevens-Johnson syndrome if carbamazepine therapy is being considered..$^{13} \mathrm{~A}$ risk evaluation and mitigation strategy (REMS) has been put in place for carbamazepine, due to the risk of cutaneous reactions and bone marrow suppression. However, the only requirement is the distribution of a medication guide to the consumer. ${ }^{14}$

\section{Oxcarbazepine}

Oxcarbazepine is structurally similar to carbamazepine and exerts its primary antiepileptic activity through its active metabolite, the 10-monohydrate derivative. Oxcarbazepine has equivalent efficacy to phenytoin, valproic acid, and carbamazepine, with typical response rates of $20 \%-30 \%{ }^{8,15-17}$ Some patients who have failed initial carbamazepine therapy have responded to oxcarbazepine. The drug is extensively metabolized, but avoids the enzymatic autoinduction of carbamazepine. It does have metabolism-based drug interactions through inhibition of CYP 2C19 and 3A4 in a dose-dependent manner, with mild induction of UDP-glucuronosyltransferase, that should be considered prior to therapy or when additional medications are added. Oxcarbazepine is almost exclusively eliminated renally as the 10-monohydrate derivative metabolite or its derivatives, necessitating renal function monitoring with accompanying dose regimen adjustments in patients with renal impairment. Therapeutic drug monitoring is not routinely done.

The most commonly reported adverse effects associated with oxcarbazepine therapy include dizziness, headache, nausea, vomiting, and fatigue. Movement or vision disturbances, upper respiratory tract infections, and nervousness are also commonly reported. A significant number of patients experience hyponatremia at a higher frequency with oxcarbazepine as compared with carbamazepine, but this is most often asymptomatic. Unspecified rashes, Stevens-Johnson syndrome, and toxic epidermal necrolysis, as well as multiorgan hypersensitivity reactions, have been rarely reported to occur soon after therapy was initiated, and patients should be monitored for such events after the start of oxcarbazepine use. ${ }^{1,10}$

\section{Phenobarbital}

Phenobarbital is a long-acting barbiturate sedative with an approximate $30 \%-40 \%$ response rate in the treatment of complex partial seizures. ${ }^{8}$ While it is not inferior to phenytoin or carbamazepine, its adverse effect profile tends to limit therapy and lead to higher patient withdrawal rates. Phenobarbital has a very slow elimination half-life, which causes significant delays to observed changes in seizure activity following dose regimen 
adjustments. Drug metabolism is extensive and variable, with significant induction of hepatic enzyme systems, resulting in numerous drug interactions. Therapeutic drug monitoring is often recommended, with a target phenobarbital range of $15-40 \mu \mathrm{g} / \mathrm{mL}$. Concentrations $>30 \mu \mathrm{g} / \mathrm{mL}$ are associated with an increased incidence of side effects.

The adverse effect profile of phenobarbital severely limits its clinical utility and often relegates it to a second-line or third-line agent in adults. Specifically, CNS depression is very common and typically leads to symptoms of sedation, drowsiness, dizziness, and lethargy. Long-term use may result in physical and psychological dependence, depression, and cognitive blunting. The possibilities of respiratory depression, hypothermia, coma, and death increase with phenobarbital concentrations $>50 \mu \mathrm{g} / \mathrm{mL}$. Hematologic, hepatic, and renal disorders have been reported, and should be evaluated through regular testing. Dermatological and connective tissue disorders have also been reported. Rare reports of severe drug hypersensitivity reactions, such as exfoliative dermatitis, Stevens-Johnson syndrome, and toxic epidermal necrolysis are possible..$^{1,10}$

\section{Topiramate}

Topiramate has demonstrated efficacy against complex partial seizures, with significant reductions in seizure frequency in $20 \%-50 \%$ of patients when used as monotherapy. ${ }^{16-18}$ Topiramate has good bioavailability and saturable but clinically insignificant protein binding. It is eliminated renally, with prominent tubular reabsorption taking place, and as such, dosing regimen should be adjusted in patients with renal impairment. Although topiramate is metabolized by both phase 1 and phase 2 reactions without significant hepatic contributions to elimination, it has been associated with metabolism-based drug interactions and should be used with caution if potential interactions are possible. ${ }^{10}$

CNS effects are the most prevalent adverse events associated with topiramate therapy, and include somnolence, dizziness, fatigue, paresthesia, and nervousness, with movement, cognitive, and psychological changes also frequently reported. Topiramate has been associated with the development of metabolic acidosis, and may be responsible for dose-dependent weight loss and anorexia following chronic use. Acute narrow-angle glaucoma and development of kidney stones are much more common with topiramate therapy compared with the incidence in the general population. Topiramate can impair sweat production and affect body temperature regulation, particularly in pediatric and geriatric patients, thus increasing the possibility of hyperthermia. ${ }^{1}$
Topiramate must be dispensed with a medication guide as part of an FDA-mandated REMS so as to educate patients on possible adverse effects. ${ }^{14}$

\section{Lamotrigine}

Lamotrigine has been shown to have a $10 \%-30 \%$ patient response rate in the treatment of complex partial seizures, with high adherence rates due in part to less severe adverse effects compared with other AEDs. ${ }^{15,17}$ Comparative studies with phenytoin and carbamazepine have shown that lamotrigine produces similar reductions in seizure activity. ${ }^{16}$ Other studies report only moderate comparative efficacy with alternative monotherapy agents. ${ }^{8}$ Lamotrigine has excellent oral bioavailability and clinically insignificant protein binding. Extensive UDP-glucuronosyltransferase-based hepatic metabolism followed by renal elimination has led to the recommendation of close monitoring for patients with severe renal or hepatic disease. Lamotrigine pharmacotherapy is sensitive to alterations in enzyme function and clearance mechanisms, with dose adjustments potentially necessary in the presence of other AEDs. ${ }^{10}$

Many of the adverse effects of lamotrigine are concentration-dependent. The most common adverse effects include dizziness, headache, weakness, rash, nausea, somnolence, and vision disturbances. Some adverse effects occur more frequently when administered with other AEDs, for example, diplopia with carbamazepine and tremor with valproic acid. Dermatological rashes are often experienced near initiation of therapy. These can be serious, are often age-related, and may be minimized through slow dose titration to therapeutic effects. ${ }^{1}$ Lamotrigine must be dispensed with a mandatory medication guide for patient education as part of a REMS. ${ }^{14}$

\section{Felbamate}

Felbamate is highly efficacious as monotherapy or adjunctive therapy in the treatment of complex partial seizures. ${ }^{19}$ However, postmarketing reports have associated felbamate therapy with aplastic anemia and acute liver failure, including some patient fatalities. ${ }^{20}$ These serious adverse effects occurred at a much higher frequency than is seen in the general population, and are estimated to be 1/3,000 for aplastic anemia and 1/10,000 for hepatic failure, with possibly higher rates in children and in those with other comorbid factors. ${ }^{1,21}$ Patients and prescribers should sign an informed consent form related to these serious adverse effects prior to initiating therapy. However, this form is not required by the FDA as part of a REMS and is kept with the prescriber. No dispensing restrictions or monitoring parameters 
are imposed by the FDA. Aside from these serious effects, felbamate is generally well tolerated, with common adverse effects of anorexia, weight loss, insomnia, nausea, and headache. Felbamate has favorable pharmacokinetic properties, but regimens should be adjusted in patients with renal dysfunction. The drug has been associated with numerous reported drug interactions that should be evaluated prior to initiation of felbamate therapy or any other drug. Therapeutic drug concentration monitoring is typically not required. ${ }^{10}$ Clinically, felbamate is considered a last-line treatment option due to possibilities of serious and potentially fatal aplastic anemia and/or acute liver failure.

\section{Adjunctive agents for complex partial seizures}

Numerous agents have been approved by the FDA for the adjunctive treatment of complex partial seizures. These include gabapentin, pregabalin, tiagabine, levetiracetam, zonisamide, lacosamide, and vigabatrin. Most studies of these agents were conducted in treatment-refractory patients who had responder rates similar to the different AEDs. ${ }^{16-18}$ However, these drugs are not easily compared to determine superiority due to different study designs and variations in inclusion criteria. Therefore, adjunctive therapeutic agent selection is typically determined by patient and provider preferences when comparing drug factors, including adverse effect profiles, clinical precautions, and possible drug interactions.

\section{Gabapentin and pregabalin}

Gabapentin and pregabalin are structurally and pharmacologically similar, with comparable adverse effect profiles. Commonly reported effects include headache, dizziness, fatigue, drowsiness, depression, psychological changes, and movement disturbances. Additional adverse effects include substantial weight gain, a high incidence of peripheral edema that might precipitate dyspnea, vision disturbances, and rare reports of hypersensitivity reactions or blood disorders. Gabapentin and pregabalin are almost exclusively eliminated renally and should be used with caution in patients with renal dysfunction. These agents have virtually no drug interactions due to the avoidance of hepatic metabolism and clinically insignificant protein binding. ${ }^{1,10}$ Both gabapentin and pregabalin have patient education-based medication guides as part of their approved labeling. The guide for gabapentin must be provided to the patient as part of a REMS for the drug. However, the REMS originally in place for pregabalin, which also required presentation of the medication guide to the consumer, was nullified by the FDA as of early 2011, because the risk of use of the drug was no longer felt to outweigh the potential benefits. ${ }^{14,22}$

\section{Tiagabine}

Tiagabine has been associated with dizziness, fatigue, drowsiness, irritability, and mood and movement disturbances, with infrequent reports of moderate to severely debilitating weakness. Tiagabine is also reported to cause cognitive impairments and stupor, with altered cortical electrical conduction. These electrical abnormalities might be indicative of altered seizure activity, and warrant special consideration for tiagabine use and avoidance of medications that lower the seizure threshold. Drug interactions with tiagabine are primarily associated with its role as a substrate for CYP 3A4. It does not inhibit or induce hepatic enzymes, nor does it interact through competitive protein binding., ${ }^{1,10}$ Tiagabine must be dispensed with a medication guide for patient education according to a REMS. ${ }^{14}$

\section{Levetiracetam}

Levetiracetam therapy is commonly associated with headache, drowsiness, sedation, weakness, movement disturbances, behavioral disturbances such as agitation or hostility, hair loss, loss of appetite, vomiting, and weight loss. Clinically significant blood disorders such as leukopenia, neutropenia, pancytopenia, and thrombocytopenia have been reported and warrant monitoring. Rare reports of pancreatitis and hepatitis have been associated with levetiracetam therapy and warrant additional monitoring. Renal function should be evaluated and appropriate dose adjustments made in patients with renal dysfunction taking levetiracetam, due to its extensive renal elimination by glomerular filtration. Levetiracetam is associated with minimal drug interactions. ${ }^{1,10}$ Patients receiving levetiracetam must receive an educational medication guide as mandated by a REMS. ${ }^{14}$

\section{Zonisamide}

Zonisamide has an adverse effect profile, much like topiramate, due in part to the presence of similar chemical moieties. ${ }^{23}$ Specifically, zonisamide is associated with sedation, dizziness, cognitive impairment, headache, vision disturbances, irritability, nausea, weight loss, and possible impairment of sweat production with accompanying changes in temperature regulation. Patients should be monitored for signs and symptoms of metabolic acidosis and be counseled to increase fluid intake to avoid the formation of kidney stones if they are susceptible. Hypersensitivity reactions are rare, 
but have been reported and include severe conditions, such as toxic epidermal necrolysis, Stevens-Johnson syndrome, fulminant hepatic necrosis, agranulocytosis, aplastic anemia, and other blood disorders. Zonisamide does not have clinically relevant competitive protein binding interactions with other drugs. It does not inhibit or induce hepatic enzymes, but is substantially metabolized by CYP 3A4 and UDPglucuronosyltransferase, and is involved in several clinically relevant drug interactions. ${ }^{1,10}$ A medication guide must be dispensed with zonisamide as part of a REMS according to FDA mandate. ${ }^{14}$

\section{Lacosamide}

Lacosamide is a newer AED that has not been included in meta-analyses or AED-based therapeutic reviews, but it has similar reported response rates as compared with other AEDs. ${ }^{24}$ It is associated with adverse CNS effects similar to other agents, with dizziness, headache, fatigue, movement disturbances, vision disturbances, and psychological changes having been reported. Lacosamide also causes nausea, vomiting, and easy bruising. There have been reports of euphoria and psychological dependence following use. Potentially serious cardiac arrhythmias have been reported, and patients should be monitored with special precautions taken if comorbid cardiac disease is present. An isolated case of delayed multisystem hypersensitivity was reported in clinical trials, and additional reports of hepatitis, hepatic dysfunction, rash, and myocarditis have also been noted. ${ }^{25}$ Lacosamide is a CYP 2C19 substrate, but does not inhibit or induce hepatic enzymes. It is extensively eliminated renally, and requires renal function monitoring and dose regimen adjustments in patients with severe renal impairment. ${ }^{1,10}$ Lacosamide must also be dispensed with a patient medication guide due to REMS' requirements. ${ }^{14}$

\section{Use of vigabatrin for refractory partial seizures}

Vigabatrin was approved in 2009 by the FDA following lengthy regulatory discussions and clinical studies, with an indication of adjunctive treatment for complex partial seizures in adults. This indication is based on numerous prospective, double-blind, placebo-controlled trials, in which its efficacy has been clearly demonstrated. ${ }^{26,27}$ Positive response rates, defined as a reduction in seizure frequency of $\geq 50 \%$, were observed in $24 \%-67 \%$ of patients. ${ }^{26,28,29}$ These robust response rates occurred consistently, despite differing inclusion criteria, varied adjunctive vigabatrin doses (although $3 \mathrm{~g} /$ day was the most common dose studied), diverse concomitant AED use, wide-ranging study lengths, and nonuniform study designs. Within these studies, subject retention rates were equivalent or superior to rates reported with other AEDs, which indicates that vigabatrin therapy is well tolerated.

Vigabatrin is rapidly and thoroughly absorbed following oral administration. It has negligible protein binding, with virtually no hepatic metabolism. Vigabatrin is eliminated primarily by the kidneys, and dosage regimen adjustments are necessary in patients with renal dysfunction. The few reported drug interactions with vigabatrin are minor, and vigabatrin does not require therapeutic adjustment when administered concomitantly with other AEDs. ${ }^{30}$

The adverse effect profile of vigabatrin requires special considerations that come with rigorous monitoring requirements. Vigabatrin has a lower reported incidence of adverse CNS effects common to other AEDs, including headache, drowsiness, sedation, and fatigue. ${ }^{31}$ Adverse psychiatric effects associated with vigabatrin therapy tend to be more prevalent than with most other AEDs, and include agitation, depression, anxiety, emotional lability, psychosis, and mania. ${ }^{32}$ However, these psychiatric disturbances are usually mild or have subtle symptomatology. Even so, they have been associated with treatment withdrawal, and patients should be monitored for such events.

There have been serious and frequent reports of idiosyncratic permanent visual field loss with vigabatrin. Because of this, vision testing prior to therapy and regularly throughout therapy is an FDA requirement. This visual field loss was originally reported to occur in approximately $30 \%$ of patients, based on controlled studies that were primarily intended to evaluate antiseizure efficacy rather than adverse effects. ${ }^{33}$ However, a recent review of all available studies involving vigabatrin therapy suggested a median incidence of $45 \% .{ }^{34} \mathrm{It}$ should be noted that up to $90 \%$ of patients who present with measurable visual field loss are asymptomatic. ${ }^{35}$ The variation in reported incidences might be attributable to differing detection methodologies, variances in patient enrollment, and nonuniform researcher reporting methods.

As part of the drug's approval process, a restrictive REMS was mandated by the FDA to monitor and track permanent visual field loss associated with vigabatrin therapy. ${ }^{14}$ This REMS requires numerous steps that must take place prior to the initiation of therapy. ${ }^{36}$ These include presentation of a patient medication guide, baseline vision testing, prescriber enrollment with accompanying education, and patient consent and education. The FDA also currently restricts the dispensing of vigabatrin prescriptions to four mail-order 
pharmacies in the US with prescription orders, refills, and third-party reimbursements facilitated through a program referred to as Support, Help and Resources for Epilepsy (SHARE). A second patient consent is required if therapy is found to be efficacious and is expected to continue after an initial trial of up to 12 weeks. ${ }^{27}$ This trial duration is considered safe because the earliest reported incidence of visual field loss in an adult is after nine months of vigabatrin use. ${ }^{37}$ The REMS also mandates quarterly testing for visual field loss, with accompanying documentation as managed by the SHARE program in order for vigabatrin therapy to continue. Finally, the REMS mandates final vision testing within 3-6 months following discontinuation of vigabatrin therapy. Ultimately, therapeutic decisions involving vigabatrin when visual field loss has occurred are made by the provider and patient. Failure to follow guidelines for regular vision testing requirements and accompanying documentation could result in eventual discontinuation of therapy as mandated by the SHARE program. This restrictive REMS is unlike that imposed on any other AED, and its implementation has resulted in dramatically increased costs for therapy in the US as compared with other countries.

\section{Comparative AED analysis}

There are only a few published AED comparisons focused on antiseizure efficacy in complex partial seizures or refractory epilepsy and they tend to be based on the systematic review of drug-focused reports or studies rather than on head-to-head comparisons in controlled clinical trials..$^{7,11,12,15-18,38-41}$ The clinical conclusions drawn from these reviews are limited and sometimes conflicting, due in part to the design, patient characteristics, comparators, and reporting variability in the original studies as well as systematic reviews. ${ }^{42}$ However, most AEDs are considered therapeutically equivalent in a broad sense when used in difficult to treat seizures. In the limited studies that have prospectively and directly compared AEDs in patients with difficult to treat seizures, the efficacy and long-term adverse effects of carbamazepine were superior to those reported for valproic acid and phenobarbital, but were approximately equivalent to phenytoin. ${ }^{43,44}$ Separate studies demonstrated that lamotrigine and tiagabine are equivalent, while levetiracetam has equivalent antiseizure activity but produces a faster response than topiramate in complex partial seizures. ${ }^{45,46}$ As previously discussed, drug antiseizure efficacy must be balanced with the potential for drug interactions, adverse effect profile, and numerous patient factors, such as renal and hepatic function. Therefore, the place of vigabatrin in the treatment of refractory complex partial seizures will be based on the balancing of clinical risks and possible patient benefits.

\section{Conclusion}

There is an extensive body of literature supporting the efficacy of vigabatrin in refractory complex partial seizures because the drug has been in clinical use outside the US since the 1980s. However, the discovery of permanent visual field loss and a high incidence of psychiatric disturbances have relegated its use far down the line within the realm of AED therapy for resistant epilepsy. Even so, vigabatrin is a welcome addition to the treatment options for refractory complex partial seizures. Prescriber and patient comfort, along with the serious adverse effects associated with vigabatrin, will ultimately impact its place in the treatment of complex partial seizures.

\section{Disclosure}

The authors report no conflicts of interest in this work.

\section{References}

1. Shorvon SD. Handbook of Epilepsy Treatment. 3rd ed. Hoboken, NJ: Wiley-Blackwell; 2010.

2. Beleza P. Refractory epilepsy: A clinically oriented review. Eur Neurol. 2009;62(2):65-71.

3. French JA. Refractory epilepsy: Clinical overview. Epilepsia. 2007; 48 Suppl 1:3-7.

4. Blume WT. Complex partial seizures. Clinical description and diagnosis. Adv Exp Med Biol. 2002;497:9-18.

5. Monté CP, Arends JB, Tan IY, Aldenkamp AP, Limburg M, de Krom MC. Sudden unexpected death in epilepsy patients: Risk factors. A systematic review. Seizure. 2007;16(1):1-7.

6. Brodie MJ. Antiepileptic drug therapy the story so far. Seizure. 2010; 19(10):650-655.

7. Treiman DM. Management of refractory complex partial seizures: Current state of the art. Neuropsychiatr Dis Treat. 2010;6:297-308.

8. Glauser T, Ben-Menachem E, Bourgeois B, et al. ILAE treatment guidelines: Evidence-based analysis of antiepileptic drug efficacy and effectiveness as initial monotherapy for epileptic seizures and syndromes. Epilepsia. 2006;47(7):1094-1120.

9. Pellock JM. Standard approach to antiepileptic drug treatment in the United States. Epilepsia. 1994;35 Suppl 4:S11-S18.

10. Rogers SJ, Cavazos JE. Epilepsy. In: DiPiro JT, Talbert RL, Yee GC, Matzke GR, Wells BG, Posey LM, editors. Pharmacotherapy: A Pathophysiologic Approach. 7th ed. Chicago, IL: McGraw Hill; 2008.

11. Tudur Smith C, Marson AG, Williamson PR. Phenytoin versus valproate monotherapy for partial onset seizures and generalized onset tonicclonic seizures. Cochrane Database Syst Rev. 2001;4:CD001769.

12. Tudur Smith C, Marson AG, Clough HE, Williamson PR. Carbamazepine versus phenytoin monotherapy for epilepsy. Cochrane Database Syst Rev. 2002;2:CD001911.

13. Loescher W, Klotz U, Zimprich F, Schmidt D. The clinical impact of pharmacogenetics on the treatment of epilepsy. Epilepsia. 2009; 50(1):1-23.

14. Anonymous. Approved Risk Evaluation and Mitigation Strategies (REMS). 2011. Available from: http://www.fda.gov/Drugs/DrugSafety/ PostmarketDrugSafetyInformationforPatientsandProviders/ucm111350. htm. Accessed May 31, 2011. 
15. Tudur Smith C, Marson Anthony G, Chadwick David W, Williamson Paula R. Multiple treatment comparisons in epilepsy monotherapy trials. Trials. 2007;8:34.

16. French JA, Kanner AM, Bautista J, et al. Efficacy and tolerability of the new antiepileptic drugs II: Treatment of refractory epilepsy. Neurology. 2004;62(8):1261-1273.

17. Beyenburg S, Stavem K, Schmidt D. Placebo-corrected efficacy of modern antiepileptic drugs for refractory epilepsy: Systematic review and meta-analysis. Epilepsia. 2010;51(1):7-26.

18. Marson AG, Kadir ZA, Hutton JL, Chadwick DW. The new antiepileptic drugs: A systematic review of their efficacy and tolerability. Epilepsia. 1997;38(8):859-880.

19. Brown WM, Aiken SP. Felbamate: Clinical and molecular aspects of a unique antiepileptic drug. Crit Rev Neurobiol. 1998;12(3): 205-222.

20. LaRoche SM. A new look at the second-generation antiepileptic drugs: A decade of experience. Neurologist. 2007;13(3):133-139.

21. Chung AM, Eiland LS. Use of second-generation antiepileptic drugs in the pediatric population. Paediatr Drugs. 2008;10(4):217-254.

22. Hertz SH. Supplemental Approval Release REMS Requirement. 2011 Available from: http://www.accessdata.fda.gov/drugsatfda_docs/appl etter/2011/021446s023,s024,022488s001,s002,s0031tr.pdf. Accessed May 31, 2011.

23. De Simone G, Scozzafava A, Supuran CT. Which carbonic anhydrases are targeted by the antiepileptic sulfonamides and sulfamates? Chem Biol Drug Des. 2009;74(3):317-321.

24. Patyar S, Medhi B. Lacosamide, a newer antiepileptic. Neurosciences (Riyadh). 2010;15(1):3-6.

25. Kellinghaus C. Lacosamide as treatment for partial epilepsy: Mechanisms of action, pharmacology, effects, and safety. Ther Clin Risk Manag. 2009;5:757-766.

26. Faulkner MA, Tolman JA. Vigabatrin in refractory complex partial seizures: The evidence of its therapeutic value. Clinical Medicine Insights: Therapeutics. 2010;2:849-856.

27. Waterhouse EJ, Mims KN, Gowda SN. Treatment of refractory complex partial seizures: Role of vigabatrin. Neuropsychiatr Dis Treat. 2009;5: 505-515.

28. Sankar R, Derdiarian AT. Vigabatrin. CNS Drug Rev. 1998;4(3): 260-274.

29. Hemming K, Maguire Melissa J, Hutton Jane L, Marson Anthony G. Vigabatrin for refractory partial epilepsy. Cochrane Database Syst Rev. 2008;3:CD007302.

30. Rey E, Pons G, Olive G. Vigabatrin. Clinical pharmacokinetics. Clin Pharmacokinet. 1992;23(4):267-278.

31. Ben-Menachem E. Vigabatrin. Epilepsia. 1995;36 Suppl 2:S95-S104.

32. Thomas L, Trimble M, Schmitz B, Ring H. Vigabatrin and behavior disorders: A retrospective survey. Epilepsy Res. 1996;25(1):217.
33. Kalviainen R, Nousiainen I. Visual field defects with vigabatrin: Epidemiology and therapeutic implications. CNS Drugs. 2001;15(3): 217-230.

34. Maguire MJ, Hemming K, Wild JM, Hutton JL, Marson AG. Prevalence of visual field loss following exposure to vigabatrin therapy: A systematic review. Epilepsia. 2010;51(12):2423-2431.

35. Wild JM, Martinez C, Reinshagen G, Harding GF. Characteristics of a unique visual field defect attributed to vigabatrin. Epilepsia. 1999;40(12):1784-1794.

36. Katz RG. Risk Evaluation and Mitigation Strategy (REMS): Support, Help and Resources for Epilepsy (SHARE). 2011. Available from: http:// www.fda.gov/downloads/Drugs/DrugSafety/PostmarketDrugSafety InformationforPatientsandProviders/UCM187533.pdf. Accessed May $31,2011$.

37. Willmore LJ, Abelson MB, Ben-Menachem E, Pellock JM, Shields WD Vigabatrin: 2008 update. Epilepsia. 2009;50(2):163-173.

38. Cramer JA, Fisher R, Ben-Menachem E, French J, Mattson RH New antiepileptic drugs: Comparison of key clinical trials. Epilepsia. 1999;40(5):590-600.

39. Muller M, Marson AG, Williamson PR. Oxcarbazepine versus phenytoin monotherapy for epilepsy. Cochrane Database Syst Rev. 2006;2:CD003615.

40. Taylor S, Tudur Smith C, Williamson PR, Marson AG. Phenobarbitone versus phenytoin monotherapy for partial onset seizures and generalized onset tonic-clonic seizures. Cochrane Database Syst Rev. 2001;4:CD002217.

41. Tudur Smith C, Marson AG, Williamson PR. Carbamazepine versus phenobarbitone monotherapy for epilepsy. Cochrane Database Syst Rev. 2003;1:CD001904.

42. Maguire MJ, Hemming K, Hutton JL, Marson AG. Overwhelming heterogeneity in systematic reviews of observational anti-epileptic studies. Epilepsy Res. 2008;80(2-3):201-212.

43. Mattson RH, Cramer JA, Collins JF. A comparison of valproate with carbamazepine for the treatment of complex partial seizures and secondarily generalized tonic-clonic seizures in adults. The Department of Veterans Affairs Epilepsy Cooperative Study No. 264 Group. N Engl J Med. 1992;327(11):765-771.

44. Smith DB, Mattson RH, Cramer JA, Collins JF, Novelly RA, Craft B. Results of a nationwide Veterans Administration Cooperative Study comparing the efficacy and toxicity of carbamazepine, phenobarbital, phenytoin, and primidone. Epilepsia. 1987;28 Suppl 3:S50-S58.

45. Chmielewska B, Stelmasiak Z. Clinical evaluation of Gabitril and Lamictal for drug-resistant epilepsy in adults. Ann Univ Mariae Curie Sklodowska Med. 2001;56:35-42.

46. Specchio LM, Boero G, Specchio N, et al. Evidence for a rapid action of levetiracetam compared to topiramate in refractory partial epilepsy. Seizure. 2006;15(2):112-116.
Therapeutics and Clinical Risk Management

\section{Publish your work in this journal}

Therapeutics and Clinical Risk Management is an international, peerreviewed journal of clinical therapeutics and risk management, focusing on concise rapid reporting of clinical studies in all therapeutic areas, outcomes, safety, and programs for the effective, safe, and sustained use of medicines. This journal is indexed on PubMed Central, CAS,

\section{Dovepress}

EMBase, Scopus and the Elsevier Bibliographic databases. The manuscript management system is completely online and includes a very quick and fair peer-review system, which is all easy to use. Visit http://www.dovepress.com/testimonials.php to read real quotes from published authors. 